rationaliste du médecin français $L$ e c on te d u $\mathrm{N}$ o üy et la théorie cosmolytique de l'anthropologue italien Alberto B lan c.

Dans son développement logique le plus général, ma propre hypothèse de l'évolution part d'un infini chaos de points géométriques, infiniment non libres et, par des univers successifs de lignes, de plans, de volumes, de vies, de pensées..., tend vers l'unicité d'un Etre Suprême qui, seul, est infiniment libre.

L'on a reproché une contradiction apparente à ce thème général, contradiction qui résulte d'une assimilation, non certaine, de la notion d'effort à la notion de contrainte. Du point de vue métaphysique, l'on peut indéfiniment discuter sur la liberté. Nos meilleures autorités ne s'en font pas faute, pour la majorité desquelles tout bonheur social est nécessairement lié à l'exact égalitarisme dont un chaos, fait de points géométriques immobiles, est le modèle idéal.

La philosophie matérialiste, qui engendre un tel idéal, repose presque toute entière sur le sophisme athéo-darwinien. Il n'est guère de naturaliste systématicien qui ne se croie tenu d'admettre ce sophisme.

Nous avons voulu, dans ces quelques phrases, montrer combien, du point de vue logique, cette obligation est fallacieuse.

Du point de vue moral - mais ceci est une autre histoire - l'on peut également juger bon d'affirmer que toute liberté nouvelle suppose un nouveau devoir et que de tout devoir nouveau naît une liberté nouvelle.

\title{
Zur Frühgeschichte der Inhalationsnarkose 1846/47
}

Von $\mathrm{H}$ a n s Fis ch er, Zürich

Das Jahr 1846 gilt in der Geschichte der Medizin allgemein als das Entdeckungsjahr der Inhalationsnarkose. Es ziemt sich wohl, an dieses Faktum nach 100 Jahren als an eine der segensreichsten medizinischen Entdeckungen, die jemals gemacht worden ist, kurz zu erinnern. Zwar wird dieses Datum, wie namentlich neuere amerikanische und englische medizinhistorische Forschungen ergeben haben, nicht ohne eine gewisse Willkürlichkeit als Geburtsjahr der Inhalationsanästhesie bezeichnet. Und doch dürfen wir, wenn wir vom praktischen Gesichtspunkt der allgemeinen Verbreitung der Inhalationsnarkose ausgehen, an dieser Jahreszahl festhalten: denn tatsächlich fällt in dieses Jahr nicht nur die Geburts- 
stunde der zu operativen Zwecken, d.h. zur Schmerzausschaltung verwendeten Inhalationsnarkose mit Äther, sondern auch die rasche Verbreitung dieses Verfahrens in allen Operationssälen der Kulturwelt.

Die Geschichte der Inhalationsnarkose, dieses wahrhaft historischen Augenblicks in der Medizin, ist so reich an Widersprüchen, Peripetien, dramatischen Zuspitzungen, unentwirrbaren Vorkommnissen, daß es sich bei Anlaß der hundertjährigen Wiederkehr dieses Ereignisses lohnt, das Augenmerk wenigstens auf einige der wichtigsten Etappen in der Frühgeschichte der Narkose zu richten. Drei Stoffe haben um die Priorität

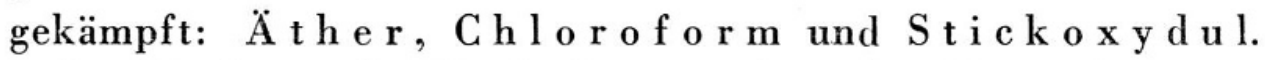

Das Verdienst der Entdeckung narkotischer Eigenschaften von Gasen und Dämpfen und die Idee ihrer Verwendung zur Ausschaltung des Operationsschmerzes gebührt zweifellos den Anglo-Amerikanern. Es war im Jahre 1772, als der englische Theologe Jos e p h Pri e s t le $y^{1}$ das Stickoxydul entdeckte. Seiner eigenartigen Wirkung wegen empfahl er es zur Inhalationsbehandlung bei Lungenaffektionen, Astmathikern usw.. Dr. Th o m a s B ed d o e s², Arzt in Bristol, ging auf diese Idee ein. So entstand im Jahre 1789 jenes berühmte medizinisch-pneumatische Institut (Pneumatic Institute) des Dr. Thomas Beddoes in Clifton, in welchem Gastanks aufgestellt waren, aus denen Patienten mit Lungenaffektionen zu therapeutischen Zwecken Stickoxydul inhalierten. Vom Jahre 1795 an ließen derselbe Beddoes und dazu der Arzt $\mathrm{Rich}$ ard $\mathrm{P}$ e a r s o $\mathrm{n}^{3}$ in Birmingham Lungenkranke zur Beseitigung oder Milderung der Atemnot auch $\AA_{\mathrm{t}}$ he $\mathbf{r} \mathrm{d}$ ä m p f einatmen. Aber noch war der wesentliche Schritt nicht getan: die schmerzbetäubende Wirkung weder des einen noch des andern Stoffes war klar erkannt. Diese Erkenntnis verdanken wir dem späteren Leiter des Beddoes'schen Institutes, dem genialen englischen Chemiker H u m p h y D a v (1778 bis

1 Joseph Priestley, Observations on different kinds of air. Phil. Trans. Roy. Soc. 62, 147 (1772).

2 Thomas Beddoes and James Watt, Considerations on the medical use of facticious airs and on the manner of obtaining them in large quantities. Bristol 1794. Watt hatte sich mit diesem Problem in der Hoffnung beschäftigt, sein kranker Sohn Gregory könne durch Gasinhalation geheilt werden. Er konstruierte eigens Apparate für die Herstellung verschiedener Gase zur Verwendung in Spitälern und im Privathaus.

${ }^{3}$ Richard Pearson berichtet in der zweiten Auflage des unter Note 2 genannten Buches in einem Brief aus Birmingham vom 2. Februar 1795 über die Wirkung des Äthers bei Lungentuberkulose, die er für sehr günstig hält. 
1829) ${ }^{4}$, welcher erstmals 1799 die betäubende und schmerzstillende Wirkung des Stickoxyduls an sich selber feststellte: in der Beddoesschen Stickoxydulkammer vergingen seine Zahnschmerzen. Davy war der erste, welcher die praktische Folgerung aus dieser Beobachtung zog, indem er schon damals das «Lachgas» zu chirurgischen Zwecken empfahl. Niemand ging jedoch auf seine Anregung ein. So vergingen wieder fast zwanzig Jahre. Dann machte ein kleiner Artikel Mi chael Fa rad a y s$^{5}$ im «Journal of Science and the Arts», 1818, darauf aufmerksam, daß die Ätherwirkung durchaus derjenigen des betäubenden Stickoxyduls gleiche. Faraday warnte ausdrücklich zur Vorsicht beim Experimentieren mit Ätherdämpfen, denn ein Gentleman sei dadurch in einen tiefen 30stündigen Schlaf verfallen, so daß man für sein Leben bangte. Die Warnung Faradays war nicht unangebracht; denn um dieselbe Zeit erzählt $\mathrm{G}$ u y d e $\mathrm{M}$ a u p a s s a $\mathbf{n} \mathbf{t}$ von einer merkwürdigen Leidenschaft, welche in Amerika um sich gegriffen hatte, der Passion zum Ätherrausch, den «ether frolics». Schließlich befaßte sich auch die experimentelle Toxikologie mit diesem Problem: der große spanische, in Paris wirkende Toxikologe $\mathrm{Orfil} \mathrm{a}^{6}$ vermochte Hunde durch Ätherinjektion und orale Ätherzufuhr regelrecht zu betäuben. Auch berichtete er in seinem «Traité de Toxicologie» von einem jungen Mann, der nach Einatmen von Ätherdämpfen bewußtlos wurde. Er blieb mehrere Stunden in einem totenähnlichen Schlaf, aus welchem er nur durch frische Luft und Anregungsmittel errettet werden konnte. Ähnliches hören wir von dem Toxikologen Ri c h a r d C h r is tis o n, Professor für gerichtliche Medizin in Edinburg, welcher in seinem Buch "A Treatise on Poisons» (Edinburgh 1829) berichtet, daß ihm zwar kein Fall bekannt sei, in

${ }^{4}$ Humphry Davy, der zwanzigjährig (1798) in das Beddoessche Institut als Assistent eintrat, studierte schon 1799 die Wirkung des Stickoxyduls an Tieren und Menschen, wobei unter letzteren sich ihm die Spitzen des literarischen Bristol, ein Samuel Taylor Colridge, Robert Southey, Tom Wedgwood u. a., zur Verfügung stellten. Über seine Erfahrungen berichtete er 1800 in dem berühmten Buch: «Researches, chemical and philosophical, chiefly concerning nitrous oxyde, or dephlogisticated nitrous air, and its respiration», by Humphry Davy, London 1800.

5 Nach John F.Fulton and Madeline E.Stanton, The centennial of surgical anaesthesia an annotated catalogue of books and pamphlets bearing on the early history of surgical anaesthesia, Henry Schuman, New York 1946, ist es nicht sichergestellt, daß diese Notiz über den Schwefeläther tatsächlich von Michael Faraday stammt.

${ }^{6}$ M. J. B. Orfila, Traité de toxicologie générale. 2 Bände. 5. Auflage. Paris 1852. 
welchem der Schwefeläther tödlich gewirkt habe; hingegen erwähnt er einen andern, in welchem ein Mann so lange Ätherdämpfe eingeatmet habe, daß er während 36 Stunden in einen Zustand von «Lethargie» und Pulsschwäche versetzt worden sei. Im übrigen beurteilt Christison den Äther in seiner physiologischen Wirkung ähnlich wie das Lachgas.

Trotz vieler solcher Einzelbeobachtungen am Menschen blieb der Äther als chirurgisches Narkosemittel noch unentdeckt.

Zunächst wurde ein ganz anderer Weg, der zur Analgesie führen sollte, eingeschlagen: Franz Anton Mesmer (1734-1815) hatte schon 1779 sein später so berühmt gewordenes Buch über den tierischen Magnetismus herausgegeben ${ }^{7}$. Doch wurde seine Idee vom magnetischen Schlaf, einer Art Hypnose oder Wachsuggestion, erst sehr viel später, dann aber mit größter Leidenschaft und Begeisterung von Laien und manchen Ärzten aufgegriffen. Wenn er sich auch der Anwendung des tierischen Magnetismus zur Unterdrückung des Operationsschmerzes gegenüber persönlich ablehnend verhielt, wurde die Idee von ärztlicher Seite bald in die Tat umgesetzt.

So machte der Pariser Chirurg J u l e s C l o q u e t 1829 eine Mammaamputation in Hypnonarkose, damals magnetischer Schlaf genannt. In England war es J a mes B raid (1795-1860) ${ }^{8}$, der in seiner "Neurypnology» 1843, also gerade in den Jahren, welche der Entdeckung der Inhalationsnarkose vorausgingen, darüber berichtet. Die Begeisterung über diese Art hypnotischer Analgesie war allerdings nicht allgemein, und kritische Geister warnten. Neben Braid hat namentlich der Schotte John Elli ot s on $(1791-1868)^{9}$ am University College Hospital in

7 Seiner Hauptschrift gingen voraus: F. A. Mesmer, «Schreiben an einen auswärtigen Arzt über die Magnetcur» (1774), «Mémoire sur la découverte du Magnétisme animal» (Paris 1776), und wurde gefolgt von «Précis historique des faits relatifs au Magnétisme animal» (Paris 1781). Vgl. auch George Rosen, Mesmerism and surgery; a strange chapter in the history of anaesthesia. J. Hist. Med. 1, No. 4, New York 1946.

${ }^{8}$ James Braid, Neurypnology or the rationale of nervous sleep considered in relation with animal magnetism. Illustrated by numerous cases of its successful application in the relief and cure of disease. John Churchill, London 1843. Braids Buch über Hypnose blieb nicht ohne Einfluß auf Charcot und die weitere Entwicklung der Hypnoselehre.

9 John Elliotson, Numerous cases of surgical operations without pain in the mesmeric state; with remarks uppon the opposition of many membres of the Royal Medical and Chirurgical Society and others to the reception of the inestimable blessings of mesmerism. Baillière, London 1843. 
London, bewußt und in Erkenntnis eines besonderen Zustandes und Verfahrens die Hypnose zur Unterdrückung des Operationsschmerzes angewandt. Die Reaktion des Publikums gegen dieses Verfahren Elliotsons war aber so heftig, daß er von seinem offiziellen Posten zurücktreten mußte. - 1845 machte J a m e s E s d a i l e (1808-1859) ${ }^{10}$, ein schottischer Arzt in Calcutta, Hypnosen an Eingeborenen worüber er 1846, also im Entdeckungsjahr der Äthernarkose - auf Grund von 161 Fällen schmerzloser Operationen in Hypnonarkose berichtete. Der Mesmerismus spukte damals in vielen Köpfen weit über Deutschland hinaus und faszinierte die medizinische Welt fast ebenso wie das Laienpublikum.

Durch den jungen englischen Arzt H e n r y H i l l H i c k m a n (18001829) ${ }^{11}$ wurde die Frage der Inhalationsanästhesie erstmals systematisch von der tierexperimentellen Seite her in Angriff genommen und das Problem der «Suspended animation» im Hinblick auf die operative Schmerzausschaltung studiert. Hickmann stammte aus der Grafschaft Shorpshire, der Heimat William Witherings, des Begründers der Digitalistherapie, und war schon mit zwanzig Jahren zum Miglied des Royal College of Surgeons of London und der Royal Medical Society von Edinburg ernannt worden. In den Jahren 1820 bis 1829 experimentierte er mit Kohlensäure und mit Stickoxydul an Tieren, um Narkose zu erzielen, wie dies aus seinen zum Teil noch erhaltenen Versuchsprotokollen hervorgeht. Als Schüler $D$ a v y s hatte er begriffen, um was es sich bei dem «Lachgas» eigentlich handelte. Hickman gab den Versuchstieren zuerst Kohlensäure, um die Atmung anzuregen, fügte dann $100 \%$ Lachgas hinzu und wartete tiefe Bewußtlosigkeit ab. Die Versuche fielen ganz eindeutig positiv aus. Die Medizinische Akademie in Paris, an welche sich Hickman mit dem Ersuchen um Genehmigung zur Erprobung der narkotischen Wirkung des Stickoxyduls am Menschen wandte, lehnte in ihrer Plenarsitzung vom 21. Oktober 1828 das Gesuch wegen Gefährlichkeit und Zwecklosigkeit ab. Ein einziger trat begeistert für ihn ein: der große Feldchirurg Napoleons, L a r e y, der sich Hickman sofort

10 James Esdaile, Mesmerism in India and its practical application in surgery and medicine. London 1846.

11 Henry Hill Hickman, A letter on suspended animation containing experiments shewing that it may be safely employed during operations on animals, with the view of ascertaining its probable utility in surgical operations on the human subject. Ironbridge 1824. 
als Versuchsobjekt anbot. Hickman kehrte resigniert und enttäuscht in die Heimat zurück und starb kurz darauf im Alter von neunundzwanzig Jahren. Seine wertvollen Versuche gerieten bald in Vergessenheit.

V e l p e a u, einer der bedeutendsten französischen Chirurgen erklärte es damals für eine Utopie zu glauben, daß man jemals schmerzlos operieren könne.

Wieder vergingen Jahre. Nachdem es der alten Welt nicht gelungen war, den praktischen Weg zur Inhalationsnarkose zu finden, sollte die Neue endlich den entscheidenden Schritt tun und auch auf diesem Gebiet Pionierarbeit leisten.

Als eigentlicher Entdecker der Lachgasnarkose gilt der Zahnarzt H o r a c e W ells (1815-1848) ${ }^{12}$ in Hartford, Connecticut (USA), der am 11. Dezember 1844 an sich selbst eine Lachgasnarkose ausführen ließ, in welcher ihm ein Zahn völlig schmerzlos gezogen wurde. Als er seine Entdeckung der Harvard Medical School in Boston Ende Januar 1845 vorführte, schrie der Patient, dem er in Lachgasnarkose eine Zahnextraktion machte, so laut bei der Extraktion, daß das Experiment mit einer völligen Blamage endete. Dabei hatte der Patient keinerlei Erinnerung an den Schmerz. Wells hatte damit, wenn auch nicht die tiefe Operationsnarkose, so doch den Stickoxydulrausch als S chmerzstillungs mit tel entdeckt. Obwohl Wells noch weiter mit Lachgas experimentierte und auch einige größere Operationen in Lachgasnarkose erfolgreich durchführte, nahm er sich seinen äußerlichen Mißerfolg so sehr zu Herzen, daß er 1848 (24. Januar) Selbstmord durch Eröffnen der Pulsadern und Äthervergiftung beging. Diese Äthervergiftung hat fast symbolische Bedeutung: denn der Äther war unterdessen als Narkosemittel zu überragendem Ansehen gelangt und hatte als Konkurrent des schwieriger zu handhabenden Lachgases diesem die Früchte seines verdienten Ruhmes genommen. Hatte sich Wells in seiner 1847 in Hartford erschienenen Abhandlung «A history of the discovery of the application of nitrous oxide gas, ether and other vapors to surgical operations» für die Priorität des Stickoxyduls eingesetzt, so trat seine Entdeckung hinter derjenigen des Äthers als Narkosemittel praktisch so völlig zurück, daß die Verzweiflung Wells' - der uns übrigens als aus-

12 Horace Wells machte die erste Mitteilung seiner Entdeckung im «Hartford Courant» vom 9. Dezember 1946. Abgedruckt bei: W.H.Archer, Life and letters of Horace Wells, discoverer of anaesthesia; chronologically arranged with an appendix J. Amer. Coll. Dent. 11, 81-210 (1944). 
gesprochener Psychopath geschildert wird - einigermaßen motiviert erscheint.

Wie wir gesehen haben, war die narkotische Wirkung des Äthers schon längere Zeit bekannt. $\mathrm{H} \mathrm{u} \mathrm{m} \mathrm{p} \mathrm{h} \mathrm{r} \mathrm{y} \mathrm{D} \mathrm{a} \mathrm{v} \mathrm{y} \mathrm{s'} \mathrm{Buch} \mathrm{«On} \mathrm{medical} \mathrm{vapors»,}$ 1831 erschienen, ist eines der ältesten wissenschaftlichen Zeugnisse, welches von der Kenntnis der narkotischen Wirkung des Äthers klar Rechenschaft gibt. Aber Davy drang mit seinem Buch auch damals nicht durch.

Der erste, welcher $\ddot{A}$ th e rnarkosen zu praktisch-operativen Zwecken ausführte, war C r awford Williamson Long (1815 bis 1878$)^{13}$ aus Jefferson in Georgia (USA). Am 30. März 1842, also zwei Jahre vor der Entdeckung von Wells, nahm er die erste Operation in Äthernarkose an Mr. James Venable vor. Bis zum Jahr 1846 verfügte er über acht gut verlaufene Fälle von Äthernarkose. Long, ein kritischer und vorsichtiger Experimentator und ein seiner Verantwortung voll bewußter Arzt, verfuhr nach den klassischen Vorbildern eines $\mathrm{H}$ a r v e y, With ering, Jenner, welche zehn bis zwanzig Jahre lang experimentierten und prüften, ehe sie ihre epochemachenden Resultate in Büchlein von vorbildlich bescheidenem Umfang bekanntgaben. Von Longs Äthernarkosen hätte man wahrscheinlich noch länger nichts gehört, wenn ihn nicht nach achtjähriger Erfahrung seine Freunde ge.drängt und er dem amerikanischen Senat davon Mitteilung gemacht hätte. Praktische Folgen ergaben sich aber auch aus dieser Entdeckung der Äthernarkose nicht.

Unterdessen hatten der Zahnarzt Willi a m Th o m a s G. Mor to n (1819-1868) $)^{14}$, ein Schüler von Horace Wells, und der Chemieprofessor

${ }_{13} C . W$. Long, An account of the first use of sulphuric ether by inhalation as an anaesthetic in surgical operations. South. Med. and Surgical Journal, Dezember 1849. - Vgl. auch H.R. Raper, A Review of the Crawford W. Long centennial aniversary celebrations. Bull. Hist. Med. 13, 340 (1943), und Crawford W. Long, the pioneer in ether anaesthesia. Bull. Hist. Med. 12, 191 (1942).

${ }_{14}$ Die erste Mitteilung über Mortons gelungene Äthernarkosen erfolgte durch Henry J. Bigelow, einen jungen Bostoner Chirurgen im «Boston med. surgic. J.» vom 18. November $1846(35,209)$ unter dem Titel: Insensibility during surgical operations produced by inhalation. - Es folgten einige kurze Mitteilungen Mortons über das von ihm Letheon genannte narkotische Gas, dessen Natur er aus Patentgründen zunächst geheimhalten wollte. Eine der ersten wissenschaftlichen Darstellungen seiner Entdeckung war sein Memorandum an die Französische Akademie: «A memoir to the Academy of Sciences at Paris on a new use of sulphuric ether, by W. T. G. Morton of Boston in the USA. Presented by M. Arrago in the autumn 
und Geologe Char les Thomas Jacks on $(1805-1880)^{15}$ bereits ihre Versuche mit der Äthernarkose veröffentlicht und waren darüber in einen mit Erbitterung geführten Prioritätsstreit geraten. Der Chemiker Jackson hatte Morton den Äther zubereitet, der wegen seines nicht unbeträchtlichen Alkoholgehalts nicht immer zu befriedigender tierexperimenteller Narkose führte. Beide wandten sich an den bekannten Chirurgen $\mathrm{J} \mathrm{o} \mathrm{h} \mathrm{n} \mathrm{C} \mathrm{olli} \mathrm{n} \mathrm{s} \mathrm{W} \mathrm{a} \mathrm{r} \mathrm{r} \mathrm{e} \mathbf{n}^{16}$, nachdem sie sich entschlossen hatten, den Versuch am Menschen zu wagen. Die erste öffentliche Demonstration der Äthernarkose fand am 16. Oktober 1846 im Massachusetts General Hospital in Boston statt: Morton, damals noch Medizinstudent, narkotisierte, Warren operierte eine Halsgeschwulst an einem Patienten namens Gilbert Abbot, was völlig schmerzlos gelang.

Morton berichtete bereits am 3. November 1846 in der medizinischen Gesellschaft von Boston über seine Entdeckung. Die Kunde von den ans Wunderbare streifenden Eigenschaften des Schwefeläthers verbreitete sich rasch durch alle größeren Städte Nordamerikas und gelangte bald nach dem Mutterlande England. J a c ob Bige low von Harvard Medical School teilte die Entdeckung seinem Freunde Dr. Fran cis $B$ o o $\mathbf{t ~ t}^{\mathbf{1 7}}$ in London und dieser seinem Freunde, dem Zahnarzt $\mathrm{J}$ a $\mathrm{m}$ e $\mathrm{s}$ R o b i n s o $n^{18}$ mit, welcher alsbald wie sein amerikanischer Kollege im

of 1847», das in französischer Übersetzung erschien: «Mémoire sur la découverte du nouvel emploi de l'éther sulfurique, par W. T. G. Morton de Boston, Etats-Unis, suivi des pièces justificatives》 (Paris 1847). - Schon vor Morton hatte sich Jackson an die Französische Akademie gewandt: «Première lettre - Boston le 13 novembre 1846», C. R. Acad. Sci. Paris, 18 janvier 1847.

15 Jackson kommt zweifellos ideell die Priorität der Äthernarkose zu, ein Problem, mit dem er sich schon 1841/42 beschäftigt hatte. In wissenschaftlicher Form hat er erst sehr viel später darüber berichtet in: «A manual of etherization: containing directions for the employment of ether, chloroform, and other anaesthetic agents, by inhalation in surgical operations, intended for military and naval surgeons, and all who may be exposed to surgical operations; with instructions for the preparation of ether and chloroform, and for testing them for impurities. Comprising also a brief history of the discovery of anaesthesia.» By Charles T. Jackson, Boston 1861. - Vgl. auch G.P. Merril and J.F. Charles Fulton, Charles Thomas Jackson, Dictionary Amer. Biogr. 9, 536 (1932).

${ }_{16}$ John Collins Warren, Etherization; with surgical remarks. Boston 1848. John Collins Warren, Inhalation of etheral vapor for the prevention of pain in surgical operations. Boston med. surgic. J. 35, 375 9. Dezember 1846.

17 Jacob Bigelow, Letter to Boott on Nov. 28. 1846. Lancet 1847, I 5.

18 James Robinson, On the inhalation of ether. Lancet, 13. Februar 1847, I 168. 
Ätherrausch kariöse Zähne schmerzlos zog. Von namhaften Ärzten erzielten Dr. L i s t o $\mathrm{n}$ am University Hospital und K e y am Guys-Hospital in London die ersten glücklichen Resultate bei größeren Operationen. Ihnen folgten bald die meisten chirurgischen Autoritäten Englands.

Prioritätsstreite zwischen Morton und Jackson - beide sehr geld- und ruhmgierig - in welche 1853 der amerikanische Senat zugunsten Mortons eingriff, - sie versuchten auf ihre «Letheon» genannte «Narkosemedizin» ein Patent zu bekommen - bildeten das unrühmliche Ende dieser Entdeckungsgeschichte der Äthernarkose: der genial-exzentrische Jackson wurde geisteskrank, er starb 1878, Morton wurde zum Trunkenbold und starb in tiefstem Elend in New York 1868.

Es war deshalb nicht unweise von Harvard Medical School, zu bestimmen, daß das in Boston zur Erinnerung an die Entdeckung der Inhalationsnarkose errichtete Denkmal keinen Namen tragen sollte. Trotz dieser dunklen Schatten, welche über der Entdeckung der Äthernarkose liegen, war man sich ihrer epochemachenden Bedeutung sofort bewußt - von diesem Tage an, dem $16.0 \mathrm{k} \mathrm{tob}$ e r 1846 , trat der Äther mit Windeseile seinen Siegeszug durch alle Kliniken der neuen und alten Welt unter ungeheurem Enthusiasmus der Zeitgenossen an. Kaum ein Jahr später wurde überall unter Äthernarkose operiert.

Wer auf dem alten Kontinent die Ätherdämpfe zuerst als Narkosemittel einführte, ist wohl nicht mehr sicher zu ermitteln. Schon am 9. Januar 1847 erzielte Dr. B o s ch in Brüssel die zur Schmerzstillung gewünschte Äthernarkose.

Am 12. Januar 1847 hielt $\mathrm{M}$ a $\mathrm{lg}$ a i $\mathrm{g} \mathrm{n} \mathrm{e}^{19}$ in Paris bereits einen Vortrag über die narkotische Wirkung der Ätherdämpfe in der Akademie. Am gleichen Tage machten $\mathrm{R} \mathrm{ou} \mathrm{x}^{20}$ im Hôtel Dieu und nach ihm V e lp e a in der Pariser Charité ähnliche Versuche, aber ohne den gewünschten Erfolg. Unterstützt vom Physiologen M a g e n d i e erklärten Roux und Velpeau in einer Sitzung der Akademie die neue Entdeckung als unerheblich, zwecklos und gefährlich. Schon nach wenigen Tagen wurden sie aber durch die nun geglückten Versuche Malgaignes eines

19 J.F. Malgaigne, Emploi de l'éther. Bull. Acad. Méd. Paris 12, 262 u. 283 (12. u. 19. Jan. 1847).

${ }_{20}$ Roux, Communication relative aux effets de l'éther introduit par la respiration. C. r. Acad. Sci. Paris 24, 89 25. Januar 1847. - Roux, Sur les effets de l'éther. C. R. Acad. Sci. Paris 24, 145 u. 168 (1. u. 8. Februar 1847). Mit Diskussion von Magendie und Velpeau. 
andern belehrt, und V e l p e a u bemerkte in derselben Akademie, nachdem er acht Tage vorher dem Äther jede praktische Bedeutung abgesprochen hatte:

«Je doutais, il y a huit jours, mais aujourd'hui je n'hésite pas à dire que c'est là une grande chose, une découverte capitale et destinée à un immense avenir.» ${ }^{21}$

$\mathrm{Zu}$ den frühen Befürwortern der Äthernarkose gehört auch L a u g i e r..$^{22}$

In Deutschland gab wahrscheinlich die «Augsburger Allgemeine Zeitung» in ihrer Nummer vom 10. Januar 1847 die erste Kunde von den Wirkungen der Ätherdämpfe. Professor $\mathrm{De} m \mathrm{~m} \mathrm{e}^{23}$ in Bern scheint unter den deutschen Ärzten als erster den Schwefeläther in Gebrauch gezogen zu haben. Er exstirpierte am 23. Januar 1847 in Bern eine Nagelwurzel bei einem sehr empfindlichen vierundzwanzigjährigen Mädchen völlig schmerzlos, ebenso eine große Drüsengeschwulst am Halse eines achtzehnjährigen Mädchens und einen Lippenkrebs bei einem sechzigjährigen Mann. An Bern schlossen sich noch im Verlaufe des Monats Januar die chirurgischen Kliniken von Erlangen ${ }^{24}$, München, Wien an.

Und hier nun geziemt es sich, der verdienstlichen Tat eines Glarner Arztes, J. J. J e n n i, zu gedenken: Schon am 5. Februar 1847 amputierte Jenni in Ennenda bei einem zweiundzwanzigjährigen, sehr geschwächten Mann den rechten Fuß unter Ätherinhalation, ohne daß der Patient Schmerz verspürte. Am 11. Februar 1847 berichtete Jenni darüber in der Neuen Zürcher Zeitung. Das Beispiel Jennis machte rasch Schule: Am 8. Februar 1847 amputierte der mit Jenni befreundete Dr. Elmer $^{25}$ in Netstal in Gegenwart Jennis einem jungen Burschen einen Oberarm in Äthernarkose, ohne daß derselbe den geringsten Schmerz

21 A.Velpeau, Communication relative aux effets de l'éther introduit par la respiration. C. R. Acad. Sci. Paris 24, 91 (25. Januar 1847). - A. Velpeau, Sur les effets de l'éther. C. R. Acad. Sci. Paris 24, 129 (1. Februar 1847).

${ }_{22}$ Laugier, Observations relatives aux effets produits par l'inhalation de l'éther sulfurique. C. R. Acad. Sci. Paris 24, 123 (25. Januar 1847). - Laugier, Nouvelles observations sur les effets de l'inhalation de l'éther pendant les opérations chirurgicales. C. R. Acad. Sci. Paris 24, 276 (22. Februar 1847).

${ }^{23}$ Demme, Hermann, geb. 28. August 1802, Altenburg (Sachsen), 1833 34 Prof. für Anatomie in Zürich (Schüler Schönleins), 1835-1865 Ordin. f. Chirurgie in Bern, dort gest. 18. Januar 1867.

${ }^{24}$ F. Heyfelder, Die Versuche mit dem Schwefeläther, Salzäther und Chloroform und die daraus gewonnenen Resultate in der chirurgischen Klinik zu Erlangen. Erlangen 1848.

${ }^{25}$ Glarner Zeitung 1847, Nr. 13 
empfunden haben soll. Am 11. Februar amputierte Divisionsarzt Dr. Engwyler $^{26}$ in St. Gallen, von Jenni angeregt, ein Mammacarzinom schmerzlos.

Jenni faßte seine Erfahrungen mit der Äthernarkose in einer im Mai oder Juni 1847 bei Friedrich Schultheß in Zürich erschienenen Abhandlung von 71 Seiten, betitelt: "Erfahrungen über die Wirkungender eingeatmeten $S$ chwefelätherdämpfe im menschlichen Organismus", zusammen. Jenni schreibt dazu im Vorwort:

«Als im Januar d. J. (1847) öffentliche Blätter die Kunde verbreiteten: es seien die kühnsten und schmerzhaftesten Operationen in Nordamerika und England mittelst Einathmens des mit athmosphärischer Luft gemengten Schwefeläthers völlig schmerzlos ausgeführt worden, und innerhalb weniger Tage in verschiedenen klinischen Instituten Frankreichs und Deutschlands die Wahrheit jener Zeitungsnovelle bestätigt wurde, war es mir sehr erwünscht, schon am 5 . Februar bei einer größeren Operation mich mit eigenen Augen von der tatsächlichen Richtigkeit überzeugen zu können. Seither habe ich das Schwefeläthergas bei 38 Personen in Anwendung gezogen, und die aus diesen Versuchen gewonnenen Resultate erlaube ich mir, wenn auch schüchtern, dem ärztlichen Publikum hiermit zur Prüfung vorzulegen.»

In seiner Abhandlung beschreibt Jenni den genauen Narkoseverlauf bei diesen 38 zwischen dem 5 . Februar und 15. Mai 1847 Operierten. Die Narkose wurde teils im Beisein der Glarner Ärzte E l m e r, H e f t i und $L \mathbf{u}$ ch s ing e r nach dem Verfahren von Malgaigne durchgeführt. Als Narkotoseur Autodidakt und ohne Literatur schildert Jenni die physiologische Wirkung der Ätherinhalation, die Stadien der Narkose, so wie wir sie heute als Initial-, Irritations- und Toleranzstadien ${ }^{27}$ bezeichnen, die Nebenwirkungen des Äthers, die Sekretionsanregung, den Hustenreiz usw.

Jenni erkennt auch richtig die Äthernarkose, obwohl ihm darüber von

${ }^{26}$ St. Galler Erzähler, 1847, Nr. 14, Beilage, p. 65.

27 Die grundlegenden Experimente von Flourens (1794-1867) waren Jenni nicht bekannt: P.J.M. Flourens, Note touchant les effets de l'inhalation éthérée sur la moelle épinière. C. R. Acad. Sci. Paris 24, 161 (8. Februar 1847). - Pierre Jean Marie Flourens, Note touchant les effets de l'inhalation de l'éther sur la moelle allongée. C. R. Acad. Sci. Paris 24, 254 (22. Februar 1847).-- P. J. M. Flourens, Note touchant l'action de l'éther sur les centres nerveux. C. R. Acad. Sci. Paris 24, 340 (8. März 1847). 
anderer Seite nichts bekannt war, als eine Intoxikation des Zentralnervensystems. Sorgfältig werden von Jenni auch Indikationen und Gegenindikationen für den Äthergebrauch angegeben. Als Gegenindikationen ergeben sich ihm: Rachenoperationen wegen der Blutungs- und Aspirationsgefahr, organische Lungen- und Herzaffektionen. Über den Äther in der Geburtshilfe verbietet ihm mangelnde Erfahrung ein eigenes Urteil.

So hatte der Äther auch in der Schweiz dank Jenni sehr früh als Narkosemittel Eingang gefunden, etwas früher als beispielsweise in Berlin, wo man sich zuerst gegen den Äther wehrte, bis D i e f f e $\mathbf{n} \mathbf{b}$ a $\mathbf{c ~ h}^{28}$ in der ersten Februarhälfte des Jahres 1847 sich des Äthers zu bedienen begann.

In die letzte Etappe der Frühgeschichte der Inhalationsnarkose treten wir mit der Entdeckung der Chlor of orm narkose. Bekanntlich erfolgte diese durch den schottischen Frauenarzt James Young S i m p s o n (1811-1870) in Edinburg. Am 4. November 1847 machte er die Entdeckung, über welche er am 10 . November in der medizinischchirurgischen Gesellschaft von Edinburg berichtete und in einer ebenso gründlichen wie kurz gefaßten Studie: «A c c o u n t of a new a na esthetic agent, as a substitutefor sulphuric ether in surgery and $\mathrm{mid}$ if ery. By J. Y. Simpson. Communicated to the Medico-chirurgical Society of Edinburgh, at their meeting on $10^{\text {th }}$ November 1847, Edinburgh 1847», die Resultate seiner Erfahrungen im Druck niederlegte ${ }^{29}$.

In dieser gleichen Sitzung der Edinburger medizinisch-chirurgischen Gesellschaft vom 1 0. Nove mbe r 1847 stellte Simpson das Gesuch an den Präsidenten, er möchte eine Kommission ernennen zur experimentellen Prüfung der Eigenschaften des Chloroforms und in der nächsten Sitzung darüber berichten. Dies wurde beschlossen und eine Kommission ernannt, welcher u. a. Robert Christis on, der hervorragende Toxikologe und gerichtliche Mediziner von Edinburg, angehörte.

In einer weiteren Studie: "Anaesthetic and ot her therapeutic properties of chlor of or me", welche ein Jahr später,

28 J. F. Dieffenbach, Der Äther gegen den Schmerz. Berlin 1847.

${ }^{29}$ Die Anregung, Chloroform, damals «Perchloride of Formyl» genannt, als Inhalationsanästhetikum zu verwenden, bekam Simpson durch den Chemiker David Waldie (1813-1889) aus Liverpool (vgl. D. Waldie, The true story of the introduction of chloroform into anaesthesia. Edingburgh 1870). 
am 11. November 1848, erschien, gab er nach sorgfältiger Prüfung am Patienten einen vortrefflichen, klaren und erschöpfenden Bericht über seine chirurgischen und geburtshilflichen Erfahrungen mit der Chloroformnarkose.

Nicht ohne Interesse ist, wie Simpson im Anschluß an seine erste am 10, November 1847 erfolgte Mitteilung seine Kollegen veranlaßte, die Wirkungen des Chloroforms an sich selbst zu prüfen. Im Sitzungsprotokoll heißt es: «Die Sitzung wurde darauf vertagt, als etwas sehr Merkwürdiges geschah: Dr. Simpson hatte jedermann eingeladen, die Wirkung des Chloroforms an sich selbst zu prüfen. Mr. Yong, ein Messerschmied, meldete sich. Etwa ein Teelöffel voll Chloroform wurde auf ein zusammengefaltetes seidenes Taschentuch ausgegossen und das Chloroform eingeatmet. Nach wenigen Atemzügen wurde völlige Unempfindlichkeit erzeugt. Beim Erwachen sagte er aus, daß er genau dieselben Empfindungen gehabt habe wie nach Ätherinhalation. Dem Beispiel folgte eine ganze Reihe weiterer Personen. Bei einem (Mr. Hunter) wurde einige Erregung hervorgerufen. Er hatte nur eine kleine Dosis eingeatmet und erklärte nachher, der Effekt sei ganz ähnlieh gewesen wie bei Lachgas. Das Tasehentuch zirkulierte von einem zumi andern und viele zeigten sich von der eigenartigen Wirkung des Chloroforms ïberrascht. Wenn man in der Runde um sich blickte, bemerkte man verschiedene Gentlemen, in einem Zustand der Empfindungslosigkeit und andere in verschiedenen Stadien offensichtlicher Vergiftung. Aber alle erholten sich rasch und erklärten, sie hätten keine unangenehmen Symptome beobachtet. Einige stellten ausdrücklich fest, die während der Chloroformierung erlebten Empfindungen seien so angenehm (delightful), gewesen, daß sie gegen eine Wiederholung nichts einzuwenden hätten.»

J a m e s Y o u $\mathrm{g}$ S i m p s o n hat nicht nur das Verdienst, das Chloroform $^{30} \mathrm{im}$ November 1847 in die Geburtshilfe eingeführt zu haben, er war

${ }^{30}$ Gegen die Einführung der schmerzlosen Geburt ereiferte sich nicht nur die Geistlichkeit ( $(\mathrm{Du}$ sollst unter Schmerzen gebären»); nicht minder groß war die Opposition eines Teiles der christlich-orthodox eingestellten Ärzteschaft. Auch gab es Frauen, welche sich Gewissensbisse machten, durch Anwendung des Chloroforms, «dieser Lockspeise des Satans», wie sich ein Geistlicher audrückte, schmerzlos geboren zu haben. Diesen Einwänden trat Simpson mit einer eigenen Schrift entgegen: «Answer to the religious objeetions advanced against the employment of anaesthetic agents in midwifery and surgery. By $J$. Y. Simpson, Edinburgh» (Dez. 1847). - Zu Simpson vgl. ferner: Anaesthetic midwifery: report on its early 
auch der erste, welcher mit dem schon Ende des Jahres 1846 von Amerika herübergekommenen Äther Narkosen an Gebärenden vornahm. Der erste Fall - es handelte sich um eine schwierige Wendung - datiert vom 19. Januar 1847. Aber schon im März konnte Simpson über eine Reihe von Äthernarkosen bei instrumentell beendigten Geburten berichten. ${ }^{31}$ In dieser Mitteilung hebt Simpson als bedeutungsvollsten Punkt hervor, daß die Wehen trotz der Äthernarkose in ihrer Frequenz und Stärke unverändert bleiben. In einem Exemplar des genannten Aufsatzes, welches er dem berühmten Gynäkologen Eduard Kaspar Jakob von $\mathrm{S}$ i e b o l d in Göttingen von Edinburg aus am 28. Februar 1847 widmete, steht der handschriftliche Eintrag:

Dear Sir,

$28^{\text {th }}$ Febr. Edinburgh.

I will feel flattered by jour kind acceptance of the following brief pamflet.

During the last month $I$ have etherized a number of ladies in private practice and during natural labour. All declare they shall have it again, if again in labour. Some 20 or 30 years hence the use will $I$ believe be general in Midwifery. The more I see of it the more I feel convenced of this.

With much esteem and respect believe me

Prof. Siebold.

$$
\text { Yours very truly }
$$

Aber es kam doch zunächst anders: Noch war das Jahr 1847 nicht zu Ende, so hatte Simpson den Äther verlassen, nicht weil er gegen seine Anwendung irgendwelche Bedenken trug, sondern weil er im Chloroform ein Mittel gefunden hatte, das er dem Äther gegenüber weit überlegen hielt, wie aus seinem bereits zitierten Aufsatz «Account of a new anaesthetic agent» hervorgeht.

Wegen seiner viel stärkeren narkotischen Wirkung verdrängte das Chloroform den Äther rasch und fast vollständig. Es gab nur wenige Städte, wie Boston und Lyon, welche dem Äther auch in der Zeit des

history and progress. By J.Y. Simpson. Edingburgh 1848. - J. D. Comrie, James Young Simpson (1811-1870). Med. Press 137, 120 (1934).

31 J.Y. Simpson, Notes on the inhalation of sulphuric ether in the practice of midwifery. Monthly J. Med. Sci. (March.) Edinburgh 1847.

32 J.Fischer, Die ersten Narkosen in der Geburtshilfe. Der Schmerz 1, 39 (1928). 
Chloroformenthusiasmus treu blieben. Aber bald kamen warnende Stimmen, wie diejenige D i r a y s, welcher 1849 einen Todesfall durch Chloroform mitteilte ${ }^{33}$.

J o h n S n ow (1813-1858), welcher die erste grundlegende wissenschaftliche Mitteilung über die Chloroformnarkose beim Menschen und über den Vergleich mit der Äthernarkose veröffentlichte, machte darauf aufmerksam, daß der Chloroformtod auf Herzlähmung zurückzuführen sei. $^{34}$ Der frühe Tod Snows, der gleichzeitig ein hervorragender Epidemiologe war, ließ diese wichtige Beobachtung wieder in den Hintergrund treten. Die praktischen Erfahrungen der folgenden Jahrzehnte zwangen aber zur Abklärung. 1864 wurde ein aus englischen Medizinern und Chirurgen zusammengesetzes Komitee mit der Aufgabe betraut, die Gefährlichkeit des Chloroforms zu untersuchen und die Ergebnisse Snows abzuklären. Sie führten zu einer vollkommenen Bestätigung von Snows Ansichten. Das Komitee empfahl ausdrücklich unter freier Luftzufuhr und nicht unter geschlossener Maske zu chloroformieren.

Die erste Anwendung eines Chloroform-Sauerstoffgemisches geht auf D u C r a y (1859) zurück. Ein weiterer wichtiger Schritt geschah: Zur Chloroformersparnis führte Alexander Crombill, Chirurg in Calcutta, einer Idee C 1 a u d e B e r n a r d s aus dem Jahre 1869 folgend, die Morphinvornarkose ein. Bald setzte dann der Umschwung ein: Um das Jahr 1890 begann man allgemein die Chloroformnarkose wieder durch die ungefährlichere Äthernarkose zu ersetzen. Mit dem Beginn des 20. Jahrhunderts war das Chloroform fast völlig aus den Operationssälen verschwunden.

Erstaunlich ist, wie wenig man sich zunächst über den eigentlichen Wirkungsmechanismus der Inhalationsnarkose Rechenschaft gab. Lange

${ }^{33}$ Der erste Chloroformtod erfolgte am 28. Januar 1848 bei Newcastle (Dr. Meggison). Die besonderen Verhältnisse deuten auf einen Herzfrühtod infolge Überempfindlichkeit (Sekundenherztod?). - Trotz der dogmatischen Behauptung Sedillots (Bull. Soc. Chir. Paris 2, 336, 1851), welcher behauptete, bei richtiger Applikation des reinen Chloroforms könne dieser Stoff selbst bei langdauernder Narkose niemals töten, kamen immer wieder Chloroformtodesfälle vor.

${ }^{34}$ John Snow, On the inhalation of the vapour of ether in surgical operations. London 1847. - John Snow, On Chlorofrm and other anaesthetics. London 1858. Die große Bedeutung Snows für die Entwicklung von Theorie und Technik der Inhalationsnarkose wird in dem ausgezeichnet dokumentierten Buch von Barbara M. Duncum, «The Development of inhalation anaesthesia», London 1947, Oxford University Press, mit Recht hervorgehoben. 
Zeit begnügte man sich damit, klinische Erfahrungen zu sammeln und entschied sich auf Grund derselben bald zum Chloroform, bald zum Äther, bald zu Mischungen beider Narkosemittel mit Alkohol. Eine eigentlich wissenschaftliche Vertiefung in das Wesen der Narkose blieb zunächst aus. In der Theorie der Chloroformwirkung wurde in einer großen Reihe von Arbeiten das Blut als der eigentliche Angriffspunkt betrachtet.

Flourens, Snow, Sedillot u. a. definierten nach dem Vorgang F a u r e s (1858) die Chloroformnarkose als eine Asphyxie, bedingt dadurch, daß das in die Lunge eindringende Chloroform das in ihr zirkulierende Blut zur Koagulation bringe und die Sauerstoffaufnahme beschränke. Andere glaubten, daß das Chloroform dem Blut direkt den Sauerstoff entziehe. Wieder andere gelangten auf Grund mikroskopischer Beobachtungen zu der Annahme, daß die Inhalationsanästhetika zerstörend auf die Blutkörperchen wirken - auch dies im Sinne einer Asphyxietheorie der Narkose, begründet auf den hämolytischen Wirkungen des Chloroforms. Dieser Ansicht war auch noch $\mathrm{O}$ swald S ch mi ed e berg (1867), welcher die narkotische Wirkung desselben auf eine Zerstörung der roten Blutkörperchen und eine Verbindung des Chloroforms mit Bestandteilen der Erythrocyten zurückführte.

All diesen in mannigfacher Variation aufgestellten Theorien trat B e rn s t e in (1870) mit dem Nachweis entgegen, daß die Narkose auch an völlig entbluteten Fröschen eintritt.

Neben dieser «Bluttheorie» der Narkosewirkung waren aber die Wirkungen der Narkotika auf das Zentralnervensystem nicht unbeachtet geblieben, welche von Flo u r e $\mathrm{n} \mathrm{s}, \mathrm{B}$ ell u. a. schon um 1847 analysiert worden waren. Auch die um 1900 dominierende Lipoidtheorie der Narkose hatte schon in der Ansicht von P a p p e $\mathrm{n}$ h e i m und G o o d (1847) ihre Vorläufer, welche besagt, daß die Äthermoleküle vermöge der Verwandtschaft des Äthers zum Fett die Funktionsfähigkeit der Nervenmoleküle affiziere und vorübergehend vernichte. $\mathrm{B}$ ib ra und $\mathrm{Har}$ $1 \mathrm{e} \mathrm{s} \mathrm{s}^{35}$ gelangten ebenfalls schon 1847 zu einer Art negativer Lipoidtheorie der Narkose, indem sie die narkotische Wirkung des Äthers auf die Herauslösung von Fetten aus dem Nervengewebe des Zentralnervensystems zurückführten. $\mathrm{L}$ a $1 \mathrm{l}$ e $\mathrm{m}$ a $n \mathrm{~d}^{36} \mathrm{u}$. a. führten die Wirkung von

${ }^{35}$ E. von Bibra und E.Harleß, Die Wirkung des Schwefeläthers in chemischer und physiologischer Beziehung. Erlangen 1847.

${ }^{36}$ L. Lallemand, M. Perrin et J.L.P. Duroy, Du rôle de l'alcohol et des anesthésiques dans l'organisme. Paris 1860. 
Alkohol, Äther, Chloroform auf die besondere Affinität dieser Stoffe zum Nervensystem und die dadurch bedingte Anhäufung im Nervengewebe zurück.

Damit sind wir bereits in die Nähe derjenigen Narkosetheorie gelangt, welche als Lipoidtheorie der Narkose von $\mathrm{H}$ a $\mathrm{s} \mathrm{H}$ ors t $\mathrm{M}$ e ye $\mathrm{r}^{37}$ in Wien (1899) und dem eine Zeit lang in Zürich wirkenden englischen Botaniker Ch arles Ernest $O$ verton (1901) für einige Jahrzehnte eine tragbare, wenn auch nicht unbestrittene Geltung besessen hat.

\title{
Anaesthetic and other therapeutic properties of chloroform ${ }^{1}$
}

\author{
By J. Y.S i m p s o n, M. D., F. R. S.E. \\ Professor of midwifery in the university of Edinburgh, and \\ Physician-accoucheur to her Majesty in Scotland, etc. \\ From the monthly Journal of Medical Science, December 1847
}

At the first winter meeting of the Edinburgh Medico-Chirurgical Society (10 $10^{\text {th }}$ November), I directed the attention of the members to a new respirable anaesthetic agent which I had discovered a short time previously, - viz., Chloroform, Chloroformyle, or Perchloride of Formyle. In this limited notice, I shall state briefly some of the principal facts pertaining to its history, composition, effects, \& c.

${ }^{37}$ H. H. Meyer, Zur Theorie der Alkoholnarkose. 1. Mitteilung. Welche Eigenschaft der Anaesthetica bedingt ihre narkotische Wirkung? Arch. exper. Path. Pharm. 42, 109 (1899).

${ }_{38}$ E. Overton, Studien über die Narkose, zugleich ein Beitrag zur allgemeinen Pharmakologie. Jena 1901.

${ }_{1}^{1}$ Die Wiedergabe dieses Originalaufsatzes von Simpson über Chloroformnarkose, eines einzigartigen und sehr selten gewordenen Dokumentes, erfolgt nach einem im Besitz der Zürcher Zentralbibliothek befindlichen Abdruck.

In diesem Zusammenhang sei auch auf den Aufsatz William Oslers: «The first printed documents relating to modern surgical anesthesia», in: Proc. Roy. Soc. Med. London 11, 65 (1917), und auf John F. Fultons: «The Morton and Warren Tracts on Ether (Letheon)», published as an appendix to: Thomas E. Keys, The history of surgical anesthesia, New York 1945, hingewiesen.

Mortons Memorandum an die Pariser Akademie vom 31. Juli 1847 wurde kürzlich durch J. F. Fulton in einem Neudruck herausgegeben: «A memoir to the academy of sciences at Paris on a new use of Sulphuric Ether by W. T. G. Morton of Boston in the USA Presented by M. Arrago in the autumn 1847. Henry Schuman, New York 1946.» (H. Fischer.) 